\title{
Towards the Next Generation of e-VLBI
}

\section{Ralph Spencer ${ }^{1}$}

The University of Manchester

Alan Turing Building, Oxford Rd., Manchester M13 9PL, UK

E-mail: resajb.man.c.uk

\section{Richard Hughes-Jones}

DANTE

City House, 126-130 Hills Road, Cambridge, UK

E-mail: Richard.Hughes-Jones@dante.org.uk

\section{Dimitra Simeonidou}

University of Essex

Dept. of Computing and Electronic Systems, Wivenhoe Park, Colchester CO4 3SQ, UK

E-mail:dsimeo@essex.ac.uk

Connected element radio interferometers currently under construction or planned will use very high data rates compared with traditional VLBI. The data rates - at multiples of 10's of Gigabits per second per antenna element - are needed to give $\mathrm{GHz}$ bandwidths at high observing frequencies where few digitisation bits are required, and to allow higher numbers of bits at low observing frequencies where the radio frequency interference (RFI) environment is aggressive. This paper describes the efforts being made to increase the current rather limited capability of eVLBI compared with connected element systems. The results of 4 Gbps tests will be discussed, and a proposal to develop 100 Gbps or higher networking will be described.

The 9th European VLBI Network Symposium on

The role of VLBI in the Golden Age for Radio Astronomy and EVN Users Meeting

Bologna, Italy

September 23-26, 2008

\footnotetext{
$1 \quad$ Speaker
} 


\section{Introduction}

The new telescopes for Radio Astronomy now under construction make full use of modern high speed digital and photonic capabilities in order to deliver bandwidth and data fidelity. ALMA and the EVLA [1,2] use $8 \mathrm{GHz}$ bandwidth in 2 polarisations, which at 3 bit digitisation results in 96 Gbps data rates, including framing overheads etc. this becomes 120 Gbps on each telescope data link. e-MERLIN [3] uses $2 \mathrm{GHz}$ bandwidth in 2 polarisations at 3 bits, though $\mathrm{L}$ band $(1.3-1.8 \mathrm{GHz})$ has $0.5 \mathrm{GHz}$ bandwidth in 2 polarisations at 8 bits, needing $30 \mathrm{Gbps}$ data links per telescope. Systems at high observing frequencies need bandwidth for full sensitivity, whereas low frequencies need more bits for RFI mitigation. The net effect is that the state of the art instruments have multi-Gbps rates on data links to the correlator. VLBI needs to have similar capability if it is to take part in the new science generated by the next generation of radio telescopes.

\section{What are we doing now?}

Current development is concentrated in the EC funded EXPReS [4] project where in the FABRIC Joint Research Activity we plan e-VLBI test observations at $4 \mathrm{Gbps}(0.5 \mathrm{GHz}$ bandwidth, 2 polarisations) from Onsala and Metsahovi to Jodrell Bank using the e-MERLIN WIDAR correlator. A 4.2 Gbps lightpath between Onsala and Jodrell Bank has been set up through SUNET in Sweden through to Copenhagen via NORDUnet and then through GEANT2 and JANET to Manchester (figure 1). A local 10 Gbps link then connects to Jodrell Bank.

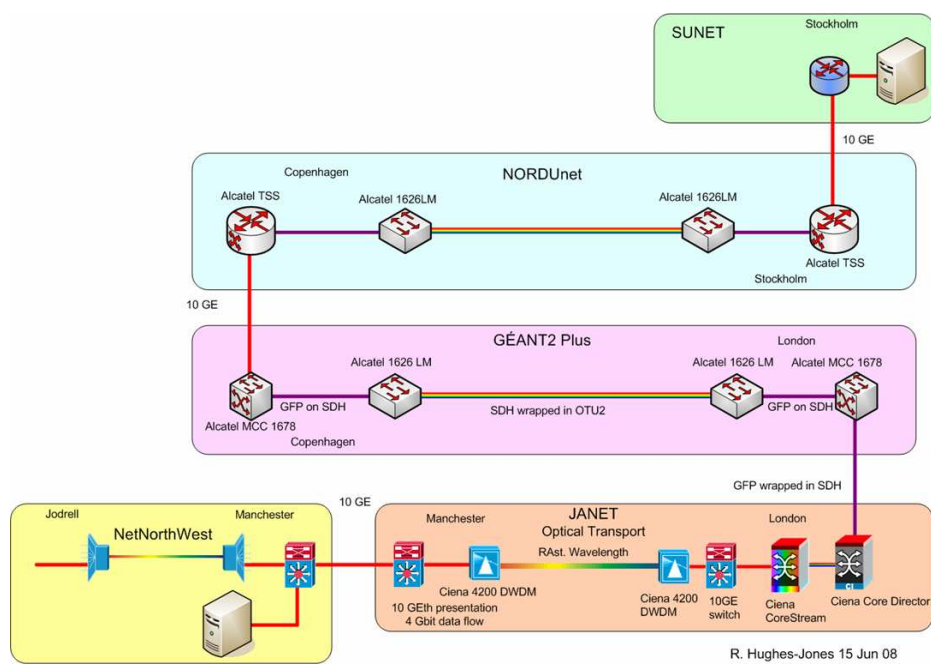

Figure 1 Test link from Stockholm to Manchester

Tests were made between PCs running Red-Hat Linux with kernel 2.6.20 running UDPmon [5] and web100 tools and equipped with Myricom 10G-PCIE-8A-R Fibre NIC cards. A maximum throughput from Stockholm to Manchester of $3.78 \mathrm{Gbit} / \mathrm{s}$ was obtained using 8192 byte frames, though with a $10 \%$ packet loss. The return path had a throughput of $4.096 \mathrm{Gbit} / \mathrm{s}$ with no packet loss. Tests have shown that the packet loss in the forward path was due to bunching of 
the packets in the transport equipment in Stockholm combined with low buffer sizes in the rest of the network. Steps are being undertaken by Alcatel to overcome this problem.

The final radio astronomy tests will be made using University of Berkeley's Internet Break-Out Boards (IBOB) [6] to take the output of the digitiser at the telescope and provide a stream of UDP packets on the 10 GE link. The IBOB receivers at Jodrell Bank then receive the 10GE packets, check and allow for packet loss or reordering, and interface to the VSI interface on the e-MERLIN correlator, currently under construction.

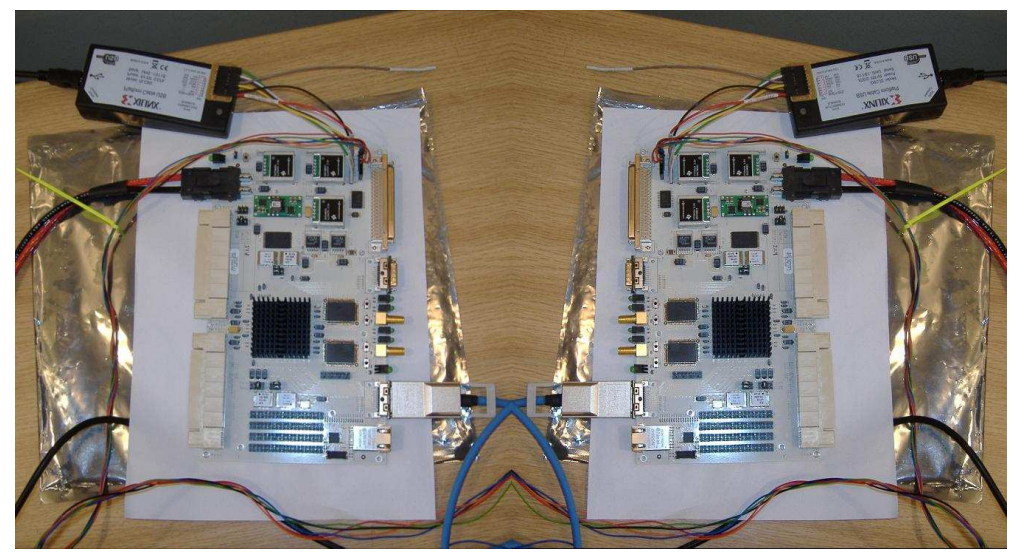

Figure 2 Berkeley's Internet Break Out Boards (IBOBs) used as Network testers and to transfer data using $10 \mathrm{GE}$ in back to back tests.

Test observations of celestial radio sources at $22 \mathrm{GHz}$ will be made, with data from the Onsala 20-m telescope correlated with those from e-MERLIN telescopes. This will result in a 4 times improvement in sensitivity over that of current VLBI observations.

\section{The Challenge of 100 Gbps plus data link system}

The advent of 100GE systems presents challenges to network providers. The international standard has been issued [7] and there is a push by network researchers and providers and to develop system which work at rates of $100 \mathrm{Gbps}$ or higher. While this may seem ambitious for e-VLBI such systems can help radio astronomy since for example SKA is expecting to generate enormous data rates of 16 Tbps or more per aperture tile station. Current optical link designs use dense wavelength division multiplexing with $10 \mathrm{Gbps}$ per wavelength, though systems with 40 Gbps per wavelength are emerging [8]. Many fibres will be needed per station for SKA, e.g. 13500 fibres carrying 430,000 channels in one design. This presents a difficult and large fibre management problem. The Universities of Manchester, Strathclyde and Essex are collaborating on a project: ADAPTNet (Application Driven Advanced Petabit Transport Networks), which will investigate Optical Time Division Multiplexing (OTDM). The idea is that multiple 10 Gbps streams will be multiplexed in time by modulating short (2 ps) pulses from a mode locked laser, resulting in $100 \mathrm{Gbps}$ or more sent on a single wavelength. Figure 3 illustrates the concepts involved in the OTDM investigation, and how it will be tested with multiple 10-Gigabit flows from iBoBs and PCs in the laboratory. This technique clearly has enormous potential for data streaming and radio astronomy applications since the all-optical networks of the future will be able to use single wavelengths for point to point transmission. 


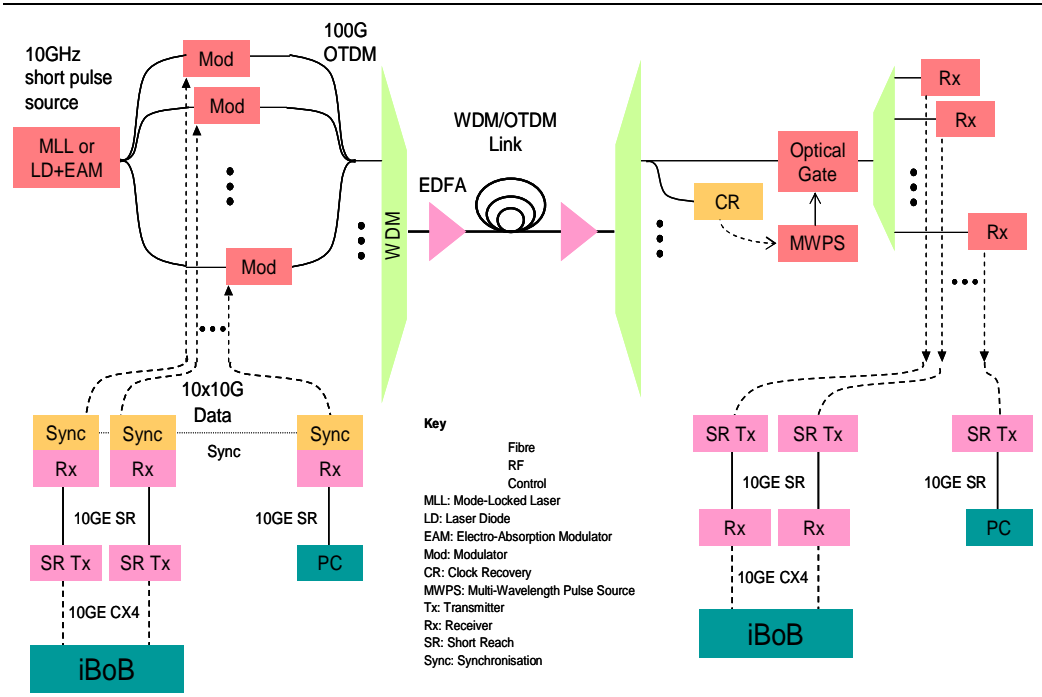

Figure 3An OTDM link will be tested in the lab using IBOB2s (the Roach) and then with the E-MERLIN correlator

\section{The Future}

The new telescopes are using very high data rates for sensitivity and fidelity of signals. If VLBI is to remain competitive, and take part in observations of the weak objects to be discovered then it needs data rates in excess of the $10 \mathrm{Gbps}$ capacity of current systems. EVN can do that if we have a next generation WIDAR type correlator capable of several $10 \mathrm{Gbps}$ per station and if a true optical path (a wavelength) can be provided through the networks.

\section{References}

[1] ALMA web page http://www.eso.org/sci/facilities/alma/, viewed Nov. 2008

[2] EVLA web page http://www.aoc.nrao.edu/evla/, viewed Nov 2008

[3] EMERLIN web page http://www.merlin.ac.uk/e-merlin/, viewed Nov 2008

[4] EXPReS web page http://www.expres-eu.org/, viewed Nov 2008

[5] On R. Hughes-Jones web page https://www.hep.manchester.ac.uk/u/rich/tools index.html, viewed Nov 2008

[6] IBOB web page http://casper.berkeley.edu/wiki/index.php?title=IBOB, viewed Nov 2008

[7] $100 \mathrm{GE}$

[8] "Sprint completes trial of 40Gbps transatlantic IP link", 25 November 2008, http://investors.sprint.com/phoenix.zhtml?c=127149\&p=irolnewsArticle_print $\& I D=1229265 \&$ highlight $=$ and http://networking.cbronline.com/news/sprint_completes_trail_of_40gbps_transatlantic_ip_link_261 $\underline{108}$ 